\title{
Parabens and Their Relation to Obesity
}

\author{
L. KOLATOROVA ${ }^{1}$, M. SRAMKOVA ${ }^{1}$, J. VITKU $^{1}$, J. VCELAK $^{1}$, O. LISCHKOVA ${ }^{1}$, \\ L. STARKA ${ }^{1}$, M. DUSKOVA ${ }^{1}$
}

${ }^{1}$ Institute of Endocrinology, Prague, Czech Republic

Received July 16, 2018

Accepted July 25, 2018

\section{Summary}

Parabens are a group of chemicals used as preservatives in the food, cosmetic and pharmaceutical industries. They are known to possess estrogenic effects, and therefore have been classified as endocrine disruptors. In addition to the classical endocrine organs, other tissues have endocrine activity, including adipose tissue. Several chemicals are known to cause obesogenic effects, and parabens are currently being studied in this context. The aim of this study was to investigate the possible connections of paraben exposure and obesity. Blood plasma from 27 healthy women was collected during their menstrual cycle. Basal anthropometric measures, levels of parabens (methylparaben, ethylparaben and propylparaben), adipokines (adiponectin, adipsin, leptin, resistin and visfatin) and hormones affecting energy balance and metabolic health (c-peptide, ghreline, GIP, GLP-1, glucagon, insulin, PAI-1) were measured. A KolmogorovSmirnov test showed higher methylparaben and propylparaben levels in women with BMI 25-34.9 compared to those with BMI 18.5-24.9. Plasma levels of methylparaben as well as the sum of parabens were positively associated with the plasma adipsin levels. Negative associations for methylparaben were found for glucagon, leptin and PAI-1. In accordance with other experimental studies we observed important associations of methylparaben and hormones affecting energy balance and metabolic health, indicating its obesogenic potential.

\section{Key words}

Paraben • Obesity • Adipokine • Endocrine disruptor • Obesogen

\section{Corresponding author}

L. Kolatorova-Sosvorova, Department of Steroids and Proteofactors, Institute of Endocrinology, Narodni 8, 11694 Prague, Czech Republic. E-mail: Ikolatorova@endo.cz

\section{Introduction}

As is generally known, there is an ongoing increase in the incidence of obesity and associated metabolic diseases in the current population. Obesity is a multifactorial and complex metabolic disease, with an etiology involving interactions between the genetic background and the environment. Poor dietary habits and lack of movement are indisputable factors contributing to the development of this disease, but the effects of the environment, stress, air pollution and smoking have also been shown to contribute to obesity (Heindel et al. 2015).

Endocrine disruptors (EDs) include thousands of chemical and natural substances that can affect the endocrine system. This biological system is based on hormone interactions, the balance of which may be disrupted by various EDs. This steroid disruption is currently the subject of much interest, as EDs usually possess estrogenic or anti-androgenic effects and their role in infertility is widely studied. EDs can act directly through the cytoplasmic steroid receptor, or also through the stimulation of estrogen synthesis. Some EDs have been shown to have the ability to affect intracellular aromatase activity, and may therefore act indirectly by increasing intracellular levels of estrogens in adipocytes with subsequent obesity potentiation in both males and females. Recent reports have shown that for successful weight reduction, in addition to dietary and daily regimens it is also advisable to avoid exposure to EDs (Darbre 2017).

"Obesogens" are generally referred to as chemicals that promote obesity by increasing the number of fat cells (and increasing fat in existing cells), by changing the number of calories burnt during resting metabolism and interfering with regulatory mechanisms 
that affect appetite and satiety. The best-known obesogen is tributyltin, used as a fungicide or wood impregnating agent. At nanomolar concentrations it binds both the $\operatorname{PPAR} \gamma$ receptor and its heterodimeric partner, the retinoid $\mathrm{X}$ receptor, and induces adipogenesis. Another group of obesogens are substances interfering with steroid receptors. Steroids are known to affect lipid storage and fat stores, and therefore this group of obesogens also plays a role in the development of adipose tissue.

In addition to the well-known tributyltin, persistent organochlorines used in the agriculture industry, bisphenol A and phthalates used in the manufacture of plastics, and parabens used as preservatives in cosmetics, pharmaceuticals and the food industry, as well as many others, have also been identified as possessing obesogenic properties (Darbre 2017). Hu et al. 2017 recently experimentally confirmed their previous observations reported in 2013 (Hu et al. 2013) that parabens promoted adipocyte differentiation in vitro and increased adiposity. The commonly used parabens are methylparaben (MP) and propylparaben (PP), which are often present in products together. Ethylparaben (EP), butylparaben and benzylparaben are others currently being used, especially in antimicrobial mixtures. It is of interest that the antimicrobial as well as estrogen activity increases proportionally to the length of the alkyl substituent. Besides estrogenic properties, some parabens have been reported to also display antiandrogenic activity by binding to androgen receptors and causing the inhibition of testosterone-induced transcription (Bledzka et al. 2014).

The combination of the antimicrobial properties and low cost and low sensitization has finally resulted in the widespread use of parabens. However, after investigating their estrogenic potential, parabens were classified as EDs, and the European Union authorized their use in only limited amounts in cosmetics, as well as in foodstuffs (Bledzka et al. 2014, Regulation EC 2009). Regarding PP, the current situation is complicated. Because of several studies documenting its negative effects on living organisms, there is still no acceptable daily intake limit (EFSA 2004, Kolatorova et al. 2017, Soni et al. 2005). Overall, according to the current legislation parabens are characterized as safe. However, on the current market, there is still increasing number of "paraben-free" products, both in cosmetics and pharmaceuticals. In the foodstuffs, as a rule consistent reading of labels is still necessary to avoid parabens.
Parabens may enter the human body through ingestion, absorption through the skin and inhalation. Their metabolism differs between these exposure pathways. Transdermal absorption is dependent on the length of the paraben alkyl- substituent, with the absorption decreasing with an increasing side chain (Andersen 2008, Vela-Soria et al. 2014). They are later metabolized by esterases, conjugated and excreted in the urine and bile. As a result of daily topical application of paraben-containing cosmetics, parabens may accumulate in the body (Mathiesen et al. 2013). Following oral exposure, parabens are metabolized by esterases in the intestine and liver, and excreted in the urine as well as in the bile and feces (Boberg et al. 2010, Vela-Soria et al. 2014).

In the present work we investigated differences between healthy women with normal (18.5-24.9) and higher BMI (25-34.9 - overweight and the first grade obesity) regarding their anthropometric parameters, plasma adipokines (cytokines secreted by adipose tissue), and other hormones affecting energy balance and metabolic health and paraben levels. We evaluated the associations between paraben exposure over one month, plasma adipokines, and levels of hormones affecting energy balance and metabolic health.

\section{Methods}

\section{Study group}

Our study included 27 healthy women aged $29.6 \pm 4.4$ (mean \pm standard deviation) years with a regular menstrual cycle (cycle length $28 \pm 3$ days), and average BMI 25.2 \pm 4.2 . The women used no medications including hormonal contraceptives and were non-smokers. Fasting samples of venous blood were taken from the cubital vein between 7:00 and 8:00 AM. Blood samples were collected in collection tubes with K2EDTA, immediately centrifuged ( $5 \mathrm{~min}, 2000 \mathrm{~g}, 4^{\circ} \mathrm{C}$ ), and plasma was stored at $-20{ }^{\circ} \mathrm{C}$ until analysis. The first sampling was performed at the start of the menstrual cycle $\left(1^{\text {st }}\right.$ or $2^{\text {nd }}$ day). Subsequent samples were then taken at regular intervals avoiding weekends and respecting the length of the menstrual cycle, for a total of 7-9 samples taken during the study. After each sample collection, the height, weight, BMI, fat (\%), fat mass, fat free mass, total body weight, waist and hip circumference were measured by anthropometric methods and by bioimpedance using a Tanita body composition monitor model TBF-310 (Tanita Corporation, Japan). The anthropometric 
characteristics of the study group are shown in Table 1.

The protocol was approved by the Ethical Committee of the Institute of Endocrinology. Informed and written consent with the use of biological materials for research reasons was obtained from all subjects participating in the project. Our study followed the Declaration of Helsinki (2000) of the World Medical Association.

Table 1. Characteristics of women participating to the study.

\begin{tabular}{lcc}
\hline Anthropometric parameters & BMI 18.5-24.9 & BMI 25-34.9 \\
\hline Age (years) & $28(26,33)$ & $28(26.8,31)$ \\
Height $(\mathrm{cm})$ & $168(164,175)$ & $170(168,175)$ \\
Weight $(\mathrm{kg})$ & $61.3(58.1,71.8)$ & $83.3(81.3,85.7)$ \\
BMI $\left(\mathrm{kg} / \mathrm{m}^{2}\right)$ & $22.2(21.3,23.5)$ & $28.5(27.8,29.6)$ \\
Fat $(\%)$ & $27.4(21.6,31.5)$ & $39(35.8,40)$ \\
Fat mass $(\mathrm{kg})$ & $16.5(12.6,20.7)$ & $32.1(30.6,34.1)$ \\
Fat free mass $(\mathrm{kg})$ & $45(43.3,48.1)$ & $50.6(49.7,53.5)$ \\
Total body water $(\%)$ & $32.9(31.8,35.4)$ & $37(36.4,39.2)$ \\
Waist circumference $(\mathrm{cm})$ & $73(69,76)$ & $82(79,90)$ \\
Hip circumference $(\mathrm{cm})$ & $100(93,109)$ & $111(106,115)$ \\
\hline
\end{tabular}

Women were divided according to BMI to two groups: normal weight women (BMI 18.5-24.9) and overweight and the first grade obesity women (BMI 25-34.9). Medians with lower and upper quartiles are given.

\section{Measurement of analytes}

The plasma levels of unconjugated parabens (MP, EP, PP) were measured using a previously published method (Kolatorova Sosvorova et al. 2017). In brief, preparation of the sample can be summarized as follows: $500 \mu \mathrm{l}$ of plasma sample was spiked with internal standards and diluted with physiological solution (1:1). Samples were extracted with tert-butyl-methylether, and the organic phase was evaporated until dryness. In the same way, an eight-point calibration was performed. The samples were derivatized using dansyl chloride in acetone and reconstituted in methanol. The sample was finally diluted 1:1 with a $10 \mathrm{mM}$ aqueous solution of ammonium formate, and $50 \mu \mathrm{l}$ was injected in the LC-MS/MS for analysis. Further analytical and validation details are reported in Kolatorova Sosvorova et al. (2017).

The plasma levels of hormones affecting energy balance and/or metabolic health (adiponectin, adipsin, c-peptide, ghreline, GIP, GLP-1, glucagon, insulin, leptin, PAI-1, resistin and visfatin) were measured using magnetic bead-based multiple assays (x-MAP technology, Luminex Corporation, Austin, TX, USA). Two kits were used: the 2-plex Bio-Plex Pro Human Adiponectin and Adipsin assay, and the 10-plex Bio-Plex Pro Human Diabetes assay (both Bio-Rad Laboratories, CA, USA). Measurements were performed on a BioPlex 200 (Bio-Rad Laboratories, CA, USA). These commercial kits were optimized before use as follows: incubation time was prolonged from one hour to two hours, and the dilution of plasma in the 10-plex kit was reduced to $1: 2$, from the recommended 1:3. Evaluation of concentrations of individual analytes was calculated by Five-Parameter Logistic Regression analysis from standard curves.

\section{Statistical analysis}

Based on conventional practice, the data that were below the limit of detection (LOD) were replaced by LOD $/ \sqrt{ } 2$ (Hornung and Reed 1990). A non-parametric analogue of the t-test, the Kolmogorov-Smirnov test, was used to explore differences in parabens and adipokines between women with normal and higher BMI. This test is performed by computing the maximum distance between the cumulative distributions of the two samples.

All data were subsequently transformed by BoxCox transformations before further processing due to the non-Gaussian data distribution and non-constant variance (heteroscedasticity) in most variables. First, partial correlation adjusted for age between anthropometric parameters and levels of parabens were examined by NCSS 2007 (Kaysville, UT, USA). Multiple linear regression analysis was subsequently used to explore associations between adipokines and parabens. Each 
model was adjusted for the same set of covariates based on statistical and biological considerations. Covariates included age, BMI, fat $(\%)$, fat mass $(\mathrm{kg})$, fat free mass $(\mathrm{kg})$, total body weight $(\%)$, waist and hip circumference $(\mathrm{cm})$ as potential confounders. All covariates were modeled as continuous independent variables. A backward stepwise selection procedure was used to construct the linear regression model. The statistical software Statgraphics Centurion XVI from Statpoint Inc. (Warrenton, VA, USA) was used for data transformations, comparisons and multiple regression analysis.

\section{Results}

In the group of women with BMI 18.5-24.9, MP was detected in $70.6 \%$, EP in $27.7 \%$ and PP in $70.6 \%$ of all samples. In the group with women with BMI 25-34.9, MP was detected in $64.6 \%$, EP in $23.3 \%$ and PP in $57.8 \%$ of all samples. The medians of MP and PP are shown in Table 2. The Kolmogorov-Smirnov test showed significant differences in the levels of MP and PP between groups with normal and higher BMI. Table 2 shows also the levels and differences in adipokine levels between these groups.

Table 2. Medians with lower and upper quartiles of parabens and adipokines in women divided into two groups according their BMI.

\begin{tabular}{|c|c|c|c|}
\hline & $\begin{array}{c}\text { BMI 18.5-24.9 } \\
\quad(n=14)\end{array}$ & $\begin{array}{l}\text { BMI 25-34.9 } \\
\quad(n=13)\end{array}$ & $\begin{array}{c}\text { Kolmogorov-Smirnov test } \\
\text { p-value }\end{array}$ \\
\hline \multicolumn{4}{|c|}{ Parabens (pg/ml) } \\
\hline Methylparaben & $255(73 ; 750)$ & $353(95 ; 1078)$ & $<0.0001$ \\
\hline Propylparaben & $59(24 ; 134)$ & $84(33 ; 157)$ & $<0.0001$ \\
\hline \multicolumn{4}{|c|}{ Adipokines (pg/ml) } \\
\hline Adiponectin & $8.72(6.53,16.3)$ & $6.62(4.62,9.72)$ & $<0.0001$ \\
\hline Adipsin & $0.569(0.496,0.718)$ & $0.548(0.481,0.671)$ & 0.485 \\
\hline Leptin & $3970(2830,5950)$ & $8980(6450,12400)$ & $<0.0001$ \\
\hline Resistin & $5650(4360,6900)$ & $5530(4240,6440)$ & 0.173 \\
\hline Visfatin & $1560(1360,2000)$ & $1380(1240,1690)$ & 0.002 \\
\hline \multicolumn{4}{|c|}{ Hormones affecting energy balance and metabolic health $(\mathrm{pg} / \mathrm{ml})$} \\
\hline C-Peptide & $793(610,942)$ & $988(766,1280)$ & $<0.0001$ \\
\hline Ghrelin & $947(659,1430)$ & $654(438,850)$ & $<0.0001$ \\
\hline GIP & $344(271,499)$ & $261(223,321)$ & $<0.0001$ \\
\hline$G L P-1$ & $375(346,474)$ & $326(298,387)$ & $<0.0001$ \\
\hline Glucagon & $405(251,499)$ & $374(335,477)$ & 0.002 \\
\hline Insulin & $279(236,342)$ & $340(235,471)$ & $<0.0001$ \\
\hline PAI-1 & $2960(2270,3620)$ & $4500(3300,6430)$ & $<0.0001$ \\
\hline
\end{tabular}

P-values indicate statistical significance, with levels less than 0.05 highlighted in bold.

In order to evaluate the relations between anthropometric measures and paraben exposure, partial correlations adjusted for age were performed. To avoid single exposures to parabens, the average month-long paraben exposure and anthropometric measures were calculated for each woman. PP and the sum of parabens ( parabens) positively correlated with hip circumference; other results of the correlation matrix are summarized in Table 3.

Multiple regressions were performed in order to investigate possible associations of parabens and adipokines. Again, to avoid single exposures to parabens, the average month-long paraben exposure was calculated for each woman. Because of the low detection of EP, sparabens was calculated from MP, EP and PP. Both plasma MP and $\Sigma$ parabens were positively associated with the plasma levels of adipsin. Opposite associations for MP were found with glucagon, leptin and PAI-1. The adjusted regression coefficients $(\beta)$ with $95 \%$ confidence intervals (CI) are summarized in Table 4. 
Table 3. Partial correlations adjusted for age between anthropometric parameters and plasma parabens.

\begin{tabular}{|c|c|c|c|c|c|c|}
\hline \multirow{2}{*}{$\begin{array}{l}\text { Anthropometric } \\
\text { parameters }\end{array}$} & \multicolumn{2}{|c|}{ Methylparaben (pg/ml) } & \multicolumn{2}{|c|}{ Propylparaben (pg/ml) } & \multicolumn{2}{|c|}{$\Sigma$ parabens (pg/ml) } \\
\hline & $\mathbf{r}$ & p-value & $\mathbf{r}$ & p-value & $\mathbf{r}$ & p-value \\
\hline$B M I\left(\mathrm{~kg} / \mathrm{m}^{2}\right)$ & 0.196 & 0.393 & 0.280 & 0.219 & 0.238 & 0.299 \\
\hline Fat $(\%)$ & 0.321 & 0.156 & 0.353 & 0.116 & 0.367 & 0.102 \\
\hline Fat mass $(\mathrm{kg})$ & 0.321 & 0.156 & 0.379 & 0.090 & 0.369 & 0.100 \\
\hline Fat free mass (kg) & 0.175 & 0.449 & 0.378 & 0.091 & 0.264 & 0.248 \\
\hline Total body water (\%) & 0.063 & 0.786 & 0.312 & 0.168 & 0.119 & 0.608 \\
\hline Waist circumference (cm) & 0.216 & 0.346 & 0.291 & 0.201 & 0.294 & 0.196 \\
\hline Hip circumference $(\mathrm{cm})$ & 0.349 & 0.121 & 0.466 & 0.033 & 0.438 & 0.047 \\
\hline
\end{tabular}

The correlation coefficients of partial correlation $r$ are a measure of the strength between variables, and the $p$-value shows statistical significance. P-values less than 0.05 are highlighted in bold.

Table 4. Adjusted regression coefficients $(\beta)$ with $95 \%$ confidence intervals (CI) for the change in plasma adipokines associated with interquartile range changes in plasma paraben concentrations.

\begin{tabular}{|c|c|c|c|c|}
\hline \multirow{2}{*}{ Adipokines } & \multicolumn{2}{|c|}{ Methylparaben } & \multicolumn{2}{|c|}{ ¿parabens } \\
\hline & $\beta(95 \%$ CI $)$ & p-value & $\beta(95 \%$ CI $)$ & p-value \\
\hline Adipsin & $0.545(0.179 ; 0.911)$ & 0.006 & $0.083(0.02 ; 0.163)$ & 0.044 \\
\hline Leptin & $-0.771(-1.424 ;-0.118)$ & 0.023 & - & - \\
\hline Glucagon & $-0.966(-1.904 ;-0.027)$ & 0.044 & - & - \\
\hline$P A I-1$ & $-0.911(-1.770 ;-0.052)$ & 0.039 & - & - \\
\hline
\end{tabular}

Each model was adjusted for the same set of covariates (age, BMI, fat (\%), fat mass (kg), fat free mass $(\mathrm{kg})$, total body weight (\%), waist and hip circumference). The models were performed using means of monthly collections of plasma samples. $\beta$ is a measure of the strength between variables and the $p$-value shows the statistical significance, with $p$-values less than 0.05 highlighted in bold.

\section{Discussion}

At the end of the last millennium, adipose tissue began to be viewed as an endocrine organ. The initial impulse was the discovery of leptin, the first of the family of hormones and cytokines secreted by adipose tissue later termed adipokines (Zhang et al. 1994). Many other adipokines such as adiponectin, adipsin, resistin and visfatin were subsequently discovered and associated with metabolic, immune, cardiovascular, neuroendocrine and reproductive systems (Bluher and Mantzoros 2015). Adipokines are generally known to be associated with controlling food intake, energy expenditure and glucose and lipid metabolism (Sramkova et al. 2015).

Leptin is important in the adaptation of the human organism to starvation, and plays a role in energy homeostasis and limiting food intake. It provides signaling of the fat and nutrient reserves, inhibits the concentration of intracellular lipids and increases glucose uptake (Bluher and Mantzoros 2015, Janeckova 2001).
The most abundant product of adipose tissue is adiponectin, which increases the oxidation of fatty acids in muscles and lowers plasma glucose, triacylglycerol and free fatty acids (Bluher and Mantzoros 2015, Haluzik et al. 2004, Nedvidkova et al. 2005). On the other hand, adipsin stimulates the transport of glucose for accumulating triglycerides in adipose cells and inhibits lipolysis (Miner 2004). Of the hormones affecting energy balance and metabolic health, glucagon is a catabolic hormone produced by pancreatic alpha cells, and enhances the conversion of adipocyte triglycerides into free fatty acids and hepatic glycogen to glucose, thus raising the glucose and fat concentrations in the bloodstream (Schade and Eaton 1974). PAI-1 (plasminogen activator inhibitor) is involved in hemostasis, the fibrinolytic system and also in atherogenesis. Its secretion was shown to be higher in visceral adipose tissue compared to subcutaneous adipose tissue, and it is known to promote unstable atherogenic plaque rupture in obese humans (Ahirwar et al. 2017). 
In this study we found significant differences in the levels of adipokines and parabens in groups of healthy women with normal and higher BMI. In the higher BMI group, higher levels of C-peptide, insulin, leptin and PAI-1 were found, which is in accordance with their physiological role in the organism. In women with normal BMI, higher levels of adiponectin, ghrelin, GIP, glucagon and visfatin were observed. The levels of resistin and adipsin did not differ significantly. Both parabens found in the majority of the samples (MP and PP) were higher in women with higher BMI, indicating their possible obesogenic activity and accumulation in fat stores. The detection rate was conversely found to be opposite. In the normal weight women all parabens were detected more frequently, however in lower concentrations in comparison with the higher BMI group. We may hypothesize, that the lower paraben levels may enter the human body via skin and higher levels via food and dietary supplements that may reflect the higher paraben levels in women with higher BMI.

To date, only few studies investigating the relations of paraben exposure and obesity are available, and moreover show inconsistent results. Recent in vitro studies reported by a U.S. group of authors show that parabens promote adipogenesis (Hu et al. 2013). In an in vitro mice experimental model, they found that parabens induce changes in gene expression related to adipocyte differentiation and lipogenesis in the adipose tissue (Hu J. et al. 2016, Hu P. et al. 2016). Based on these results, the group suggests that parabens may play role in the development of obesity. Quiros-Alcala et al. (2018) recently reported cross sectional associations between markers of adiposity and urinary paraben concentrations in a large sample of U.S. adults and children. However, they reported inverse associations between paraben exposure and adiposity markers.

Artacho-Cordon et al. (2017) recently reported the presence of MP in human adipose tissue, but that MP quantified in adipose tissue did not correlate with the concentration of MP in the urine. These results demonstrate that parabens are deposited in the human fat stores, and may help explain the contrary results of in vitro studies and studies investigating paraben exposure from urine. Considering such paraben accumulation in the fat stores, it seems likely that persons with higher fat stores may excrete lower amounts of paraben metabolites to the urine. In our study, we determined parabens directly from plasma, which should better reflect the real paraben exposure than urine. In addition, to avoid single paraben exposures, the associations were calculated from average month-long exposure.

Studies investigating the relation with parabens and adipokines are very scarce. Boberg et al. (2008) investigated the impact of parabens on insulin, leptin, PAI-1 and other adipokine levels in rat experimental models. They found that butylparaben is a weak ligand of the PPAR receptor and that butylparaben causes reductions in plasma leptin levels. In accordance with this, we observed a negative association of plasma MP and leptin levels in humans. The authors also found no association between parabens and PAI-1. However, we found a negative association in humans. Franklin et al. (2011) examined in vitro and in vivo metabolic actions of low molecular weight GIP receptor modulator 4-hydroxybenzoic acid 2-brombenzylidene hydrazide $(4 \mathrm{H} 2 \mathrm{BH})$, a paraben derivative, on mice experimental model. They found that $4 \mathrm{H} 2 \mathrm{BH}$ effectively annuls GIP activity in vitro and in vivo, with slightly less potency against glucagon action. Analogous results were observed in our study, where a negative association of MP and glucagon was found.

Contrary to glucagon, leptin and PAI-1, we observed positive associations of MP and $\Sigma$ parabens with adipsin. Adipsin was found to be identical with factor D in the alternative complement pathway in both mice and humans, and the circulating concentration of adipsin tends to correlate positively with the degree of adiposity (Pomeroy et al. 1997). The observed positive association in our study correlates with the physiological role of adipsin, and shows that parabens may potentially contribute to the increase of adipsin levels and therefore also obesity. The possible obesogenic potential of parabens was also confirmed by the positive correlations between PP and $\Sigma$ parabens with hip circumference. It could therefore be also hypothesized that paraben exposure could lead to a "healthier" body fat distribution. Moreover, observed correlations of MP with several hormones and PAI-1 may also favor a "healthier" metabolic profile.

We are aware of the limitation of our study consisting in the sample number and the absence of more sophisticated methods of body composition assessment instead of bioimpedance (e.g. dual X-ray densitometry). The future validation study will be relevant. On the basis of our results, it would be of interest to extend the study group and to include men. 


\section{Conclusion}

This pilot study is one of the few studies investigating the possible role of parabens in obesity. In accordance with other mainly experimental studies, we observed important associations of MP and adipokines indicating its obesogenic potential. In summary we observed negative associations of MP with glucagon, leptin and PAI-1 and positive associations with adipsin and $\Sigma$ parabens. The associations were observed predominately for MP, reflecting its more widespread use. This study highlights that the usage of parabens in various food, cosmetic and pharmaceutic products may be connected not only with hormone dependent health problems but also with diseases of civilization like obesity. On the other hand, the positive correlations between PP and $\Sigma$ parabens with hip circumference may point to the "healthier" body fat distribution in paraben exposed overweight and first grade obesity women.

\section{Conflict of Interest}

There is no conflict of interest.

\section{Acknowledgements}

This study was supported by MH CZ - DRO (Institute of Endocrinology - EÚ, 00023761). The research team kindly acknowledges David W. Hardekopf for the proofreading.

\section{References}

AHIRWAR AK, SINGH A, JAIN A, KAIM K, BHARDWAJ S, PATRA SK, GOSWAMI B, BHATNAGAR MK, BHATTACHARJEE J: Association of prothrombotic adipokine (plasminogen activator inhibitor-1) with TSH in metabolic syndrome: a case control study. Horm Mol Biol Clin Investig 34: 1868-1891, 2017.

ANDERSEN FA: Final amended report on the safety assessment of methylparaben, ethylparaben, propylparaben, isopropylparaben, butylparaben, isobutylparaben, and benzylparaben as used in cosmetic products. Int $J$ Toxicol 27: 1-82, 2008.

ARTACHO-CORDON F, ARREBOLA JP, NIELSEN O, HERNANDEZ P, SKAKKEBAEK NE, FERNANDEZ MF, ANDERSSON AM, OLEA N, FREDERIKSEN H: Assumed non-persistent environmental chemicals in human adipose tissue; matrix stability and correlation with levels measured in urine and serum. Environ Res 156: 120-127, 2017.

BLEDZKA D, GROMADZINSKA J, WASOWICZ W: Parabens. From environmental studies to human health. Environ Int 67: 27-42, 2014.

BLUHER M, MANTZOROS CS: From leptin to other adipokines in health and disease: facts and expectations at the beginning of the 21st century. Metabolism 64: 131-145, 2015.

BOBERG J, METZDORFF S, WORTZIGER R, AXELSTAD M, BROKKEN L, VINGGAARD AM, DALGAARD M, NELLEMANN C: Impact of diisobutyl phthalate and other PPAR agonists on steroidogenesis and plasma insulin and leptin levels in fetal rats. Toxicology 250: 75-81, 2008.

BOBERG J, TAXVIG C, CHRISTIANSEN S, HASS U: Possible endocrine disrupting effects of parabens and their metabolites. Reprod Toxicol 30: 301-312, 2010.

DARBRE PD: Endocrine disruptors and obesity. Curr Obes Rep 6: 18-27, 2017.

EFSA: Opinion of the Scientific Panel on Food Additives, Flavourings, Processing Aids and Materials in Contact with Food on a request from the Commission related to para hydroxybenzoates (E 214-219). EFSA J 2: 1-26, 2004.

FRANKLIN ZJ, MCDONNELL B, MONTGOMERY IA, FLATT PR, IRWIN N: Dual modulation of GIP and glucagon action by the low molecular weight compound 4-hydroxybenzoic acid 2-bromobenzylidene hydrazide. Diabetes Obes Metab 13: 742-749, 2011.

HALUZIK M, PARIZKOVA J, HALUZIK MM: Adiponectin and its role in the obesity-induced insulin resistance and related complications. Physiol Res 53: 123-129, 2004.

HEINDEL JJ, NEWBOLD R, SCHUG TT: Endocrine disruptors and obesity. Nat Rev Endocrinol 11: 653-661, 2015.

HORNUNG RW, REED LD: Estimation of average concentration in the presence of nondetectable values. Appl Occup Environ Hyg 5: 46-51, 1990. 
HU J, RAIKHEL V, GOPALAKRISHNAN K, FERNANDEZ-HERNANDEZ H, LAMBERTINI L, MANSERVISI F, FALCIONI L, BUA L, BELPOGGI F, TEITELBAUM SL, CHEN J: Effect of postnatal low-dose exposure to environmental chemicals on the gut microbiome in a rodent model. Microbiome 4: 26, 2016.

HU P, CHEN X, WHITENER RJ, BODER ET, JONES JO, POROLLO A, CHEN J, ZHAO L: Effects of parabens on adipocyte differentiation. Toxicol Sci 131: 56-70, 2013.

HU P, KENNEDY RC, CHEN X, ZHANG J, SHEN CL, CHEN J, ZHAO L: Differential effects on adiposity and serum marker of bone formation by post-weaning exposure to methylparaben and butylparaben. Environ Sci Pollut Res Int 23: 21957-21968, 2016.

HU P, OVERBY H, HEAL E, WANG S, CHEN J, SHEN CL, ZHAO L: Methylparaben and butylparaben alter multipotent mesenchymal stem cell fates towards adipocyte lineage. Toxicol Appl Pharmacol 329: 48-57, 2017.

JANECKOVA R: The role of leptin in human physiology and pathophysiology. Physiol Res 50: 443-459, 2001.

KOLATOROVA L, DUSKOVA M, VITKU J, STARKA L: Prenatal exposure to bisphenols and parabens and impacts on human physiology. Physiol Res 66 (Suppl 3): S305-S315, 2017.

KOLATOROVA SOSVOROVA L, CHLUPACOVA T, VITKU J, VLK M, HERACEK J, STARKA L, SAMAN D, SIMKOVA M, HAMPL R: Determination of selected bisphenols, parabens and estrogens in human plasma using LC-MS/MS. Talanta 174: 21-28, 2017.

MATHIESEN L, ZURI G, ANDERSEN MH, KNUDSEN LE: A proposed study on the transplacental transport of parabens in the human placental perfusion model. Altern Lab Anim 41: 473-482, 2013.

MINER JL: The adipocyte as an endocrine cell. J Anim Sci 82: 935-941, 2004.

NEDVIDKOVA J, SMITKA K, KOPSKY V, HAINER V: Adiponectin, an adipocyte-derived protein. Physiol Res 54: 133-140, 2005.

POMEROY C, MITCHELL J, ECKERT E, RAYMOND N, CROSBY R, DALMASSO AP: Effect of body weight and caloric restriction on serum complement proteins, including Factor D/adipsin: studies in anorexia nervosa and obesity. Clin Exp Immunol 108: 507-515, 1997.

QUIROS-ALCALA L, BUCKLEY JP, BOYLE M: Parabens and measures of adiposity among adults and children from the U.S. general population: NHANES 2007-2014. Int J Hyg Environ Health 221: 652-660, 2018.

REGULATION (EC) No 1223/2009 of the European Parliament and of the Council of 30 November 2009 on cosmetic products. $O J$ L 342: 59-209, 2009.

SCHADE D, EATON RP: Role of insulin and glucagon in obesity. Diabetes 23: 657-661, 1974.

SONI MG, CARABIN IG, BURDOCK GA: Safety assessment of esters of p-hydroxybenzoic acid (parabens). Food Chem Toxicol 43: 985-1015, 2005.

SRAMKOVA M, DUSKOVA M, VITKU J, VCELAK J, MATUCHA P, BRADNOVA O, DE CORDEIRO J, STARKA L: Levels of adipokines and some steroids during the menstrual cycle. Physiol Res 64 (Suppl 2): S147-S154, 2015.

VELA-SORIA F, RODRIGUEZ I, BALLESTEROS O, ZAFRA-GOMEZ A, BALLESTEROS L, CELA R, NAVALON A: Simplified matrix solid phase dispersion procedure for the determination of parabens and benzophenone-ultraviolet filters in human placental tissue samples. J Chromatogr A 1371: 39-47, 2014.

ZHANG Y, PROENCA R, MAFFEI M, BARONE M, LEOPOLD L, FRIEDMAN JM: Positional cloning of the mouse obese gene and its human homologue. Nature 372: 425-432, 1994. 